\title{
A Study of the Energy Saving and Capacity Improvement Potential of Power Control in Multi-hop Wireless Networks
}

\author{
Jeffrey P. Monks ${ }^{\dagger}$, Jean-Pierre Ebert ${ }^{\ddagger}$, Adam Wolisz ${ }^{\ddagger}$, and Wen-mei W. Hwu ${ }^{\dagger}$ \\ † University of Illinois Urbana-Champaign, IL 61801, USA \\ \{jmonks, hwu\}@crhc.uiu.edu \\ ₹ Technical University Berlin, Telecommunication Networks Group, \\ Sekr. FT5-2, 10587 Berlin, Germany \\ \{ebert, wolisz\}@ee.tu-berlin.de
}

\begin{abstract}
This study investigates the potential of using transmission power control in wireless packet networks with differing number of hops between source and destination. Here we exploit the benefits of power control in the context of multi-hop wireless ad hoc type networks with a distributed media access control. For our investigations we choose several general ad hoc network topologies and studied the effect of power control with respect to energy consumption and network capacity. We show that power control largely improves the network capacity and energy savings in all investigated scenarios, and that utilizing a greater number of intermediate hops between base source and destination improves the energy savings, but often causes a tradeoff in capacity, depending on the network topology scenarios.

Keywords-Power Control, Energy Saving, Multi-hop Wireless Networks, Network Capacity
\end{abstract}

\section{INTRODUCTION}

It has been shown that controlling the transmission power can offer many benefits in performance. These benefits include both capacity improvements and energy savings. This paper presents a study of capacity improvements and energy savings and tradeoffs of power control in a multi-hop wireless packet network.

Power control in cellular networks has been researched heavily [1], [2] in past years, and many techniques such as those applied in CDMA (code division multiple access) networks are currently being used in practice. More recently, researchers demonstrated with both theoretical studies [3] and simulations [4], [5] that integrating power control into ad hoc type wireless packet networks can provide considerable benefits in capacity and energy consumption. These networks, unlike cellular networks, operate without support of fixed infrastructure. A set of wireless nodes that are distributed over a localized area constitute an ad hoc network if they forward each others traffic such that nodes that are out of range can communicate through intermediate nodes. These systems generally utilize a single shared channel whose access is regulated in a distributed manner by a medium access control (MAC) protocol that is integrated into each wireless node. Considerable past work has focused on tuning the MAC protocols to maximize the channel utilization. However, more recently new protocols were proposed that integrate power control into the MAC layer for further capacity enhancement and their benefits were presented with the aid of simulation tools $[6],[7]$.

In this paper, we take the wireless ad hoc type power controlled protocol framework and extend it to evaluate the performance of multi-hop wireless networks. Utilizing multiple intermediate hops can provide extensive energy savings since transmission signals attenuate on the order of $1 / d^{4}$ in typical networks. Adding power control to the multi-hop case can provide further savings in energy. As stated above, power control has been shown to provide significant benefits in performance for single hop wireless ad hoc networks with distributed medium access control though it is of interest to see what benefits exist in a multi-hop scenario. The goal of this paper is not to define mechanisms that integrate power control into multi-hop wireless packet networks, but rather to evaluate the tradeoffs and benefits of using multiple hops between source and destination, where each node controls its transmission power based on the topology. Therefore, a generic powercontrolled MAC protocol will be presented in Section III$B$ and evaluated against the non-power-controlled IEEE 802.11 protocol [8] for various ad hoc network topologies.

The generic power-controlled protocol (GPC, we define the specifics of later) operates in the single shared channel environment and follows the collision avoidance framework. Therefore, we use the basic protocol framework defined in [7] which is composed of a radio frequency (RF) power negotiation phase and a data transmission phase. That is, new transmitters send with less power than would cause a corruption of an ongoing transmission at a neighboring receiver (collision avoidance). Furthermore, each mobile must send with the minimal power needed to reach its destination during the data transmission phase (plus a little more as a safety margin). If both of the criteria can be satisfied then the data is transmitted. If these criteria cannot be satisfied then the node backs off until such a time when the network conditions allow the constraints to be met.

The structure of this paper is as follows. Section II starts by motivating power control from both the potential energy savings and capacity improvement and suggests additional motivation for utilizing intermediate hops. Then Secti- 


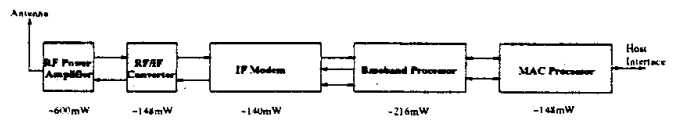

Fig. 1. Schematic WLAN network interface card and the respective typical power consumption of the components assuming the 11 $\mathrm{Mbit} / \mathrm{s}$ version of the PRISM I chipset

on III describes a power controlled and a non-power controlled wireless ad-hoc MAC protocols. In Section IV several network topology scenarios are defined in which the MAC protocols are compared. It also suggests examples of how these scenarios may be realized in a real network environment. The performance measure as well as the experimental setup are introduced in Section $\mathrm{V}$ and the specified network topology scenarios are evaluated in Section VI. Finally, section VII summarizes the paper and presents concluding remarks.

\section{Motivating RF OUtPut Power Control}

In this section, transmission power control is motivated from two prospective benefits: energy savings and capacity improvement. It is then show how reducing the transmission range so that more intermediate hopes are used can add further improvements. The energy savings is achieved by minimizing the average transmission power. It is shown below that the transmission power level is directly related to the power consumption of the wireless network interface. The second benefit is accomplished by using the network resources more efficiently. That is, by allowing a greater number of simultaneous transmissions, power control increases the total network capacity. Both of these issues are investigated further in the following two sections.

\section{A. Energy consumption}

The mobile battery lifetime is becoming an increasingly important issue to manufacturers and consumers, as mobile devices are being used more frequently in our everyday lives. Wireless network interfaces such as the ones used in a wireless LAN (WLAN) can consume a significant energy amount of a mobile device. The power amplifier, as compared to other wireless network interface components, consumes a considerable portion of the network interface power. The power consumed by the power amplifier is directly proportional to the strength (power) of the transmitted signal. Therefore, it is becoming of great interest to control the transmission power level such that the lifetime of mobile terminals is maximized. As an example, consider Fig. 1, which shows a schematic and typical power consumption levels of the WLAN network interface card utilizing the PRISM $111 \mathrm{Mbit} / \mathrm{s}$ chipset (see [9]). Notice that the power amplifier may take more than three times the power of any other individual component and consume almost half the total energy consumed by the network interface card assuming that all components are in operation. This ratio is expected to continue to increase for future

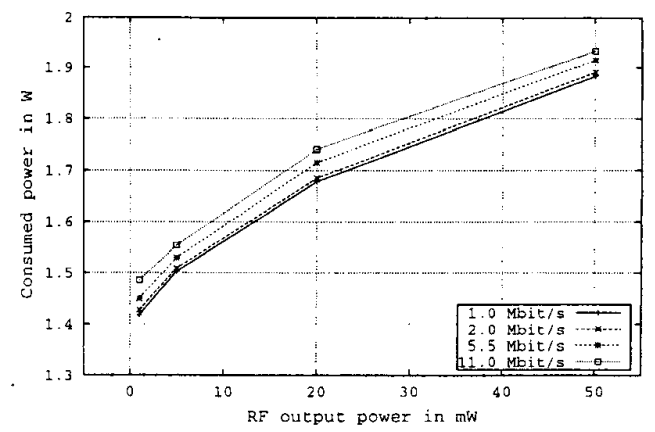

Fig. 2. Overall power consumption of an Aironet PC4800 PCMCIA interface for different RF output power levels and transmission rates during the send phase

WLAN interfaces cards, as the processing components become more power efficient and power saving technics such as on/off-switching are implemented. Therefore we believe, that there is a considerable energy savings potential in controlling the RF output power. Fig. 2 indicates how controlling the RF output power influences the power consumed by the Aironet PC4800 PCMCIA WLAN interface during the send phase. We gathered the data by measuring the voltage drop across a small resistor in line with a WLAN network interface card operating in ad hoc mode. The current can be calculated by dividing the voltage by the resistance. By multiplying the voltage drop across the network interface card and the current we have the amount of instantaneous power consumed by the WLAN interface card. The measurements were repeated for various $R F$ power levels. (see [10]).

It can be concluded from Fig. 2 that changes in the transmission power level have a large affect on the instantaneous power consumption. The higher the RF output power the higher the power consumption of the WLAN interface. In fact, an increase in the RF output power level leads to an over-proportional increase in the total power consumed by the WLAN interface. Our results show that the increase from 1 to $50 \mathrm{~mW}$ in RF output power leads to increase of about $500 \mathrm{~mW}$ in the overall power consumption. Furthermore, the graph reveals that a change of the coding scheme (transmission rate) has an almost negligible effect on the power consumption. While the the coding scheme is directly related to the throughput, it has little effect on the instantaneous power consumption. Power control, on the other hand, has a direct effect on both the throughput and energy savings, and is therefore an issue that deserves careful consideration.

To obtain a better idea of the degree of reduction in transmission power (and therefore energy savings) that power control can provide, we must look at the basic path loss model since this dictates the relationship between the transmission range and the required transmission power. 


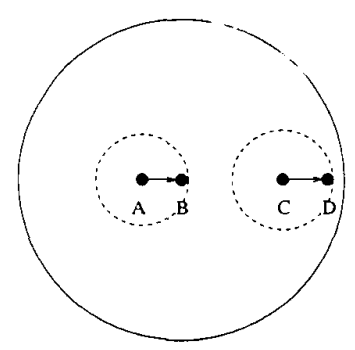

Fig. 3. Capacity enhancements observed with transmission power control

The path loss typically causes the signal to attenuate with distance on the order of $1 / d^{\alpha}[11]$, where $d$ represents distance between transmitter and receiver, and $\alpha$, the path loss factor, is typically between 2 and 6 . As a result, modest differences in transmission ranges will result in considerable differences in required transmission power to maintain the same signal quality (power level at the receiver).

Achieving the desired power savings may require that a source use one or more intermediate hops to reach its destination. As shown above, since the power needed to reach a destination increases considerably more than linearly with the distance, the use of multiple hops can be beneficial with respect to power consumption in spite of additional intermediate transmissions.

\section{B. Capacity}

As outlined in Section I, we focus here on a network model, where mobile nodes communicate on a single shared channel and without the assistance of a fixed supporting infrastructure, an ad hoc environment. Previous MAC protocols have been presented for such a network [12], [13], [8], [14] that used fixed transmission power levels. While these protocols allow transmission without collisions in the sender and receiver neighborhoods, they also limit the amount of spectral reuse since they are designed to work with fixed transmission power levels. Therefore, if we consider a case like the one demonstrated in Fig. 3, we observe that with the contemporary MAC protocols the transmission from $A$ to $B$ would prevent $C$ from sending to $D$ since it is range of $B^{1}$. However, if $A$ reduced its transmission power level to be just enough to reach $B$, and likewise $C$ would send with only enough power to reach $D$ both transmissions could happen simultaneously could therefore provide extensive increases in capacity. These benefits have been demonstrated with both theoretical studies [3] and simulations [4] that integrate power control into ad hoc type wireless networks with distributed medium access control. Further, MAC protocols were presented in [7] and [6], where considerable gains in throughput were shown.

\footnotetext{
${ }^{1}$ Many protocols use additionally the RTS/CTS mechanism as defined in [12] to avoid the effects of hidden terminal scenarios. In the picture, B would respond with a CTS to an RTS of A preventing the transmission from $C$ to $D$ even if $C$ could not hear $A$.
}

In addition to controlling the transmission power such that it is just enough to reach the destination, it is also of interest to investigate the capacity of multi-hop networks. As mentioned in the previous section, adding additional hops between the source and destination can considerably reduce the total power consumption. Therefore, it is also desirable to look at the potential benefit or cost of employing multiple hops in terms of throughput. This along with other related issues are discussed in Section IV and the corresponding results demonstrated in Section VI.

\section{Wireless Multihop Ad hoc Medium ACCESS Control Protocols}

In this section we present two MAC protocols for wireless multihop ad hoc networks which are compared in terms of capacity and energy expenditure later. The responsibility of a Medium Access Control (MAC) protocol is the arbitration of accesses to a shared medium among several end systems. As non-power controlled MAC we chose IEEE 802.11. An generalized power controlled MAC (GPC) which is defined below serves as a power controlled counterpart. The later operates by having perfect knowledge about the network and it demonstrates the potential benefits of integrating power control into the MAC protocol.

\section{A. IEEE 802.11}

IEEE 802.11 is an Ethernet-like distributed mechanism referred to as Carrier Sense Multiple Access / Collision Avoidance (CSMA/CA). Since wireless LANs lack the capability of collision detection, a collision avoidance (CA) mechanism tries to minimize access conflicts a priori.

In general if the channel is free, then a node with a packet to send senses the channel for a period of time and then transmits if the channel is free after. If the channel is busy or becomes busy during sensing, the MAC packet transmission has to be postponed until the channel becomes free and an additional waiting time has elapsed, during which the radio channel must remain free. Each node that wishes to transmit then backs off for a random interval and the first node whose backoff interval ends (the one with the shortest interval) acquires the channel.

A packet transmission can be preceded with an RTS/CTS control message exchange whereby the packet sender sends an RTS according to the aforementioned $\mathrm{MAC}$ rules and the intended receiver answers by a CTS. Thereby the sender and the receiver announce to their neighbors that a packet transmission will start. Only if the exchange of these control messages was successful the data packet transmission will start. The main objective of this mechanism is to avoid collisions in hidden terminal scenarios as well as reducing the collision phase when transmitting long packets, since only the tiny control messages can collide. The RTS/CTS message exchange should not be used for short packets since this would add unnecessary overhead. The RTS threshold value determines for what packet sizes the RTS/CTS message exchange should 
be used.

The IEEE 802.11 MAC protocol uses an immediate acknowledgment (ACK) to recover from transmission errors. After a successful data packet reception, an ACK is transmitted, indicating the correct reception. If the reception of a packet was not successful no ACK will be sent from the receiver and as a result the sender will retransmit the packet. The retransmission are performed until the data packet was received correctly or the maximum number of retransmissions is reached.

\section{B. $G P C$}

Instead of defining the mechanisms for a specific power controlled MAC protocol GPC outlines a power controlled $\mathrm{MAC}$ protocol framework whose results can then be applied to most power control implementations for the shared channel infrastructureless (ad hoc) networks. The GPC protocol is provided with perfect (global) knowledge of the link gain between any two nodes, the noise at any potential destination, and the upper bound on a transmitter's signal power needed to protect other receivers (maximum transmission power that neighboring receivers can tolerate). The protocol, like IEEE 802.11, follows the RTS-CTSDATA-ACK message exchange. However, all messages are sent with only enough power needed to reach the destination. GPC backs off if the destination requires more power than a neighboring node can tolerate. It also starts with in initial overcompensated transmission power instead of making power adjustments through the transmission since in a multiple access environment the later is not practical due to contention delay. That is the sender transmits with a specified amount more than is actually need to reach the destination. In this case, GPC demonstrates the upper bound on the performance of transmission power controlled protocols for the multiple access environment.

\section{Network Topology Scenarios}

This section outlines several network topologies which we evaluate later in terms of energy consumption and network capacity using the non-power-controlled IEEE 802.11 protocol ${ }^{2}$ and the aforementioned power-controlled GPC protocol, respectively. The network topology dictates how nodes communicate (their communications hierarchy) and the placement of certain nodes. We considered ad hoc networks of two main types. One main type is referred to as a non-clustered ad hoc network, where all nodes within the network take part in every type of network function such as relaying of packets. The second main type is referred to as a clustered ad hoc network. In a clustered ad hoc network nodes are grouped into clusters with designated forwarding agents relaying packets between clusters.

The motivation for implementing forwarding agents is to reduce the complexity of the routing algorithm and take

${ }^{2}$ We assume, that the IEEE 802.11 protocol operates in the Distributed Coordination Function (DCF) mode. advantage of nodes with greater capacity and energy resources. It has been demonstrated [15] that the overhead associated with ad hoc routing algorithms can account for more than $50 \%$ of the total packets sent in the network (depending on the average number of hops between source and destination and the mobility of mobile nodes). Using specific nodes in a cluster for forwarding packets can greatly reduce the amount of routing overhead (number of packets required to establish and maintain routes) since the routing discovery would then only require that packets be sent to the forwarders (as appose to every nodes in the network). This would reduce the complexity of the routing algorithm to the order of the number of clusters, instead of the order of the number of nodes in the network. Also if we consider a network consisting of heterogeneous mobile nodes (with differing available resources) such as cell phones, PDAs, laptops, vehicles, and fixed access points it would be advantageous to use the nodes that had greater resources to send over longer distances.

Another factor to consider for forwarding agents is whether their position can be controlled. Depending on the type of network, the position of the forwarding agents may or may not be able to be controlled. If the network can control the position of the forwarding agents, they can be placed such that any nodes distance to the forwarder is upper bounded or can be placed in accordance with the mobile density in certain areas.

The different types of networks considered here are evaluated in Section VI in terms of their energy savings and capacity improvement or tradeoff. Each of the network scenarios evaluated is now defined and an example is given to show how they apply to real network.

- Scenaria I-Non-clustered ad hoc networks: This type of network assumes that all nodes have equal resources and routing is computed in a totally distributed fashion. That is any node can be a forwarder so the routing requires that some sort of control packets be sent between every reachable node to find the best route. An example of this would be a sensor network where every node is equal (has equal resources). An advantage of such an algorithm is that every possible route is considered such that every source-destination pair is provided with the shortest route. However, as stated above such an algorithm would imply significant overhead if there is even a modest amount of mobility in the network since routes will often become disconnected so that new ones must be found.

- Scenario II - Clustered ad hoc networks with forwarding agents whose positions can be controlled: Here nodes are classified into clusters that form around designated forwarding agents (based on locality). The placement of these forwarders can be controlled to provide coverage and reachability. Nodes within a cluster can communicate directly while nodes in different clusters have to make use of forwarding agents. In Section V-B this scenario is tested with uniform place- 
ment, where the distance between forwarders is such that full connectivity is maintained. An example of such a paradigm is one where dedicated wireless nodes are actively placed to support a set of users with PDAs (personal digital assistants). Further, we investigate two cases within this scenario. The first case is where the power resources of the forwarding agents is unlimited such as a vehicle or a node with a fixed power supply, and the seconds case is where the energy of the forwarding agents is limited such as a mobile powered by batteries.

- Scenario III - Clustered ad hoc networks with forwarding agents whose positions are not controllable: This scenario is similar to the last except that the location of the forwarding agents can not be controlled and are random. Such a configuration may result if we consider vehicles or other nodes with greater resources that have other purposes than to purely serve as supporting infrastructure for mobile nodes with more limited resources. An example of this paradigm may be where a public safety officer's hand held radio communicates through the closest public safety vehicle, which would then relay the corresponding packets to other vehicles, and then to the intended receiver. One problem with this scenario is that outages may have to be tolerated since the placement of forwarding agents is random and may be out of range of the mobile nodes or other forwarding agents. Also like the previous scenario we consider forwarding agents that do and do not have limited power resources.

The performance of these scenarios is evaluated in Section VI) in terms of energy consumption and network capacity for a non-power-controlled MAC, IEEE 802.11, and a generalized power-controlled MAC, GPC.

\section{Performance Measures and Simulation ENVIRONMENT}

In this section we present the performance measures and the simulations assumptions needed to evaluate the nonpower controlled and power controlled MAC protocols.

\section{A. Performance Measures}

The above presented scenarios are evaluated in terms of network capacity and consumed signal energy per successful sent bit. The network capacity $C^{3}$, given in Equation 1 , is the number of successful transmitted bits per second within the network ( $\mathrm{Thr}$ ) normalized by the corresponding transmission rate of 2Mbit/s (TX_Rate).

$$
C=\frac{\text { Thr }}{\text { TX_Rate }}
$$

Signal energy refers to the transmission power level of each packet times the duration of the packet summed over

\footnotetext{
${ }^{3} \mathrm{C}$ is referred to as network capacity since it denotes the maximum
} achievable throughput for a certain network topology scenario. the total number of packets sent (successfully and unsuccessfully) multiplied by the total time transmitting for all nodes. The signal energy per successfully transmitted bit can then be expressed as

$$
E_{\text {bit_succ }}=\frac{\sum_{i=1}^{K} \mathrm{Pt}_{\mathrm{i}} T_{\mathrm{i}}}{\sum_{j=1}^{S} L_{\mathrm{j}}},
$$

where $K$ represents the total number of packets sent (including control packets) for the duration of that simulation (from all nodes including intermediate hops), $S$ is the total number of successful transmitted packets between source and destination, $P t_{\mathrm{i}}$ is the RF transmission power of packet $i, T_{\mathrm{i}}$ the time to transmit packet $\mathrm{i}$ and $L_{\mathrm{j}}$ is the number of payload bits of the ith successfully sent packet. Note that $\mathrm{Pt}_{\mathrm{i}} T_{\mathrm{i}}$ is the energy used to send a given packet (or packet energy). The total number of successful transmitted packets, $S$, are calculated from source to destination and not between intermediate hops, while the packet energy is summed over all packet transmissions. Therefore, it accounts for the aggregate energy used by all hops to send a bit to the final destination so that the single hop and differing number of multi-hop cases can then be fairly compared.

The RF signal energy expenditure is used here instead of the total energy used by the network interface because we want to show the particular merit of power control with respect to capacity and energy saving. Furthermore, accounting for total energy usage would require that we make assumptions about the power consumption levels of each of the components in the network interface card (as shown in Fig. 1). This would make the measurements only relevant for a particular device. Rather, we wish to measure just the energy of the transmitted signal (which is related to the average transmission power level) such that these results are relevant for any current or future wireless network interface cards. The signal energy is divided by the number of successfully sent bits so that the overhead incurred as a result of retransmissions is taken into account.

\section{B. Simulation Environment and Parameter Values}

To evaluate the performance of these MAC protocols in the different network topologies scenarios, the $n s^{2}$ simulator was used with the CMU wireless extensions [16]. The data rate for this configuration was set to $2 \mathrm{Mbit} / \mathrm{s}$, the packet size was fixed to $2 \mathrm{~KB}$, the overcompensation was set to be $2 \mathrm{~dB}$, the transmission power range for GPC was between $-5 \mathrm{dBm}$ and $22 \mathrm{dBm}$, the fixed transmission power level of 802.11 was $20 \mathrm{dBm}$ at maximum. Notice, the maximum power level of GPC was set to be $2 \mathrm{~dB}$ above the fixed power level of 802.11 by the overcompensation. The fixed range for 802.11 and the maximum transmission range for GPC were setup such that source-destination pairs could be a maximum distance of 500 meters apart to receive a valid data packet. 
The amount of RF transmission power required to send a valid signal to its destination depends on the gain between source and destination, which models the attenuation of the transmitter power over the distance. We used a simple path loss model as given in [11]. As long as the sender-receiver distance is within the Freznel zone we assumed that the signal attenuates proportional to $\frac{1}{d^{2}}$. For distances outside the Freznel zone we assumed a path loss proportional to $\frac{1}{d^{4}}$. Also some thermal noise $(-104 \mathrm{dBm})$, a receiver sensitivity of $-64 \mathrm{dBm}$ and a receive threshold of $-60 \mathrm{dBm}$ are assumed. A signal is assumed to be valid if the signal-to-interference-ratio (SIR) is at least $10 \mathrm{~dB}$.

The nodes for these scenarios (100 for these experiments) were uniformally sprinkled in a 350 meter by 350 meter network. Note with this network size can reach the furthest possible destination (in opposite corners) if the transmission power is set to the maximum range (500 meters).

For the traffic model we assumed some number of sourcedestination pairs which were picked randomly (according to a uniform distribution) off-line. When the simulation started these sources would generate packets according to independent Poisson processes. The results below are for 100 flows (source-destination pairs) generating 16 packets per second. This rate of packet generation is to keep the sources busy such that the nodes in the areas where spectral reuse can be exploited have packets to send.

For the multi-hop case, a simple off-line routing algorithm is implemented that chooses the route requiring the fewest number of intermediate hops (i.e. shortest path) for the given range settings. We note that this may be suboptimal with respect to intermediate hops for a power controlled protocol since we would ideally also want to take into account spectral reuse. However, this simple method ensures that the same routes were used for both 802.11 and GPC so that we can neglect the effects of the routing algorithm and keep the results comparable.

We want to investigate the energy and throughput performance for differing number of hops (or transmission ranges) between source and destination, and also comparing non-power-controlled and power-controlled MAC protocols for these differing transmission ranges. Therefore, simulations results were gathered for nodes having maximum transmission ranges of $500,250,166,125,82$, and 62.5 meters. That is to say, the RF transmission power for IEEE 802.11 simulations was fixed (not power controlled) to the given range, while for the GPC simulations the power level was based only on the power needed to reach the end destination. For Scenario I these ranges correspond to a maximum number of hops of $1,2,3,4,6$, and 8 , respectively, based on the network size ( 350 by 350 meters) that nodes are distributed within.

For Scenario II the corresponding maximum hops are 1, $4,6,8,10,12$ since the packets can only be relayed through the forwarding agents if source and destination do not belong to the same cluster. For Scenario III the forwarding agents are placed randomly. This means that connectivity

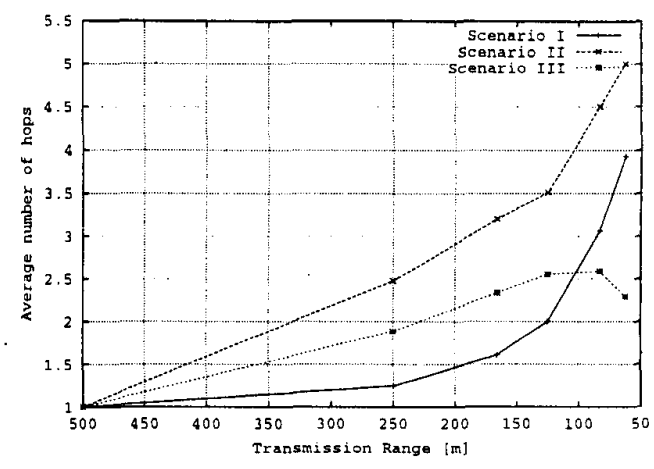

Fig. 4. Average number of hops between source-destination pairs having different transmission ranges for both GPC and IEEE 802.11 simulations

cannot be guaranteed unless the transmission range covers the entire network area ( $500 \mathrm{~m}$ or the diagonal of the 350 by $350 \mathrm{~m}$ network). Therefore, for this case the maximum transmission range is fixed to 500 meters and the number of forwarding agents is set to be the number that corresponds to those needed to ensure full coverage in Scenario II, uniform node placement (see Section VI). For Scenario I and II the results are demonstrated as the maximum number of hops between source and destination increase or transmission ranges decrease. These are the respective maximum hops and of course the averages will be less than this since the source-destination pairs are chosen at random from the nodes in the network area. The corresponding averages are shown in Fig. 4 for the basic topologies. Note that in Scenario III the maximum hops between forwarding agents is at most one since the transmission range covers the entire network area, as discussed above. Also the forwarding agents themselves can also have traffic so average hops decreases slightly when the number of forwarding agents becomes a significant portion of the total nodes. This trend is an artifact of the network topology and the communications pattern and can be observed in Fig. 4, where the average hops for the Scenario III curve drops slightly for the greatest number of forwarding agents (corresponding to the $62.5 \mathrm{~m}$ range shown on the $\mathrm{x}$-axis). This situation is not visible in Scenario I and II since there the forwarding agents still have to make use of other forwarding agents (limited transmit power) to send data to their respective destination.

\section{Performance of TOPOlOgy Scenarios}

Following the structure introduced in Section IV, we start by investigating energy saving potential and network capacity of 802.11 and GPC for a non-clustered ad hoc network, Scenario I. The performance is shown as the transmission range is decreased and therefore requiring a greater number of average hops (see Fig. 4)

Fig. 5 shows the energy savings as the transmission range is decreased. As we anticipated in Section II, the- 


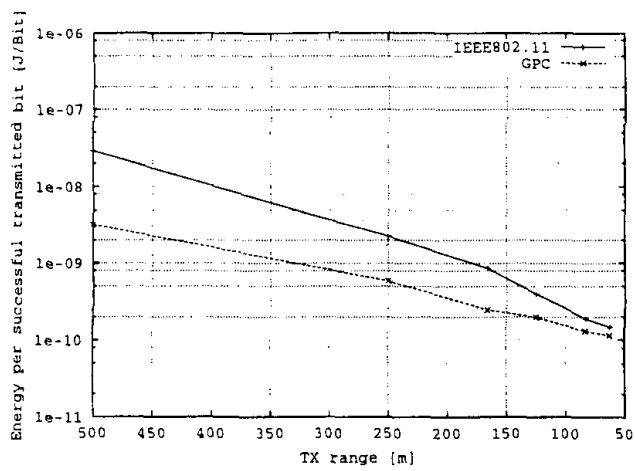

Fig. 5. Signal energy per successfully transmitted bit for an infrastructureless network with different transmission ranges

re is a significant savings in energy as the transmission range is decreased. The power controlled MAC protocol, GPC, shows additional improvement over the non-powercontrolled MAC protocol, 802.11. However, the amount of improvement decreases with transmission range. This is because as the maximum transmission range decreases the range over which the transmission power can be varied between a near and far destination also decreases (because of the use of intermediate hops) limiting the benefits of power control.

Fig. 6 now shows the throughput for 802.11 and GPC for the same ranges as the energy savings figure was presented. First observe, that the throughput of IEEE 802.11 at 500 meter starts below $100 \%$ while the throughput for GPC is slightly above. This is because every node is in transmission range of any other node in the IEEE 802.11 case which allows only for a single transmission at any point in time. Otherwise, a collision would result. However, GPC can take advantage of spectral reuse since it adapts its transmission power to the level needed to reach the intended receiver. This behavior can also be observed in the throughput results for other scenarios presented below. Observe secondly, the throughput actually drops as the number of hops is increased (transmission range is decreased). This may be counter intuitive if we refer to the theoretical analysis presented in [3] since as the range is halved the maximum hops increases by a factor of two, but the area of the transmission decreases by a factor of four. Conceptually this should allow for more simultaneous transmissions than the cost of requiring additional time slots to send the packet to the destination. However, this analysis does not take into account the fact that packet flows with multiple hops can only be sent at the rate allowed by the slowest (highest contention) link. The analysis of this theoretical study assumes that each link is able to send packets independent of the last hop (i.e. that each hop has a sufficient number of packets buffered that it has packets to send when the network area becomes free). This is obviously not the case in a true scenario, where only the slowest link can take

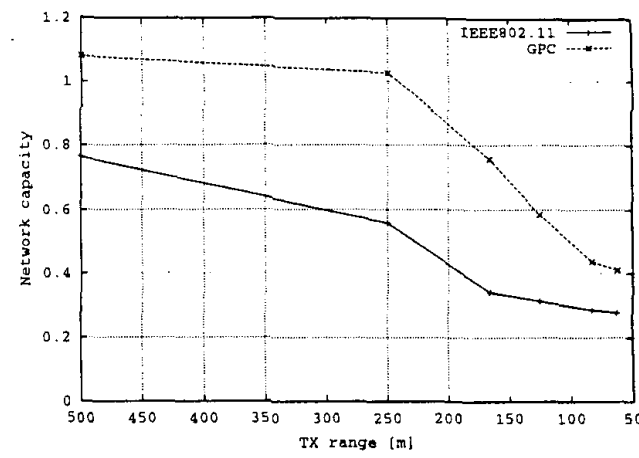

Fig. 6: Normalized throughput for an infrastructureless network with different transmission ranges

full advantage of the extra spectral reuse offered by the decreasing transmission ranges.

Another characteristic to note is that the throughput curves do not decline smoothly with decreasing transmission range. Particularly, the transition from $500 \mathrm{~m}$ to 250 $m$ is more gradual than the next. This is because even at $250 \mathrm{~m}$ most nodes are still within one hop of each other since only nodes at opposite corners are actually $500 \mathrm{~m}$ apart. Furthermore, nodes near the center are in range of all other nodes. Therefore, the throughput drops more gradually than the later ranges, where a greater number of nodes require multiple hops to send packets to their destination.

The benefits in throughput (number of packets successfully delivered) provided by power-controlled over nonpower-controlled MAC protocols are decreased as the range is reduced for the same reason the energy benefits of power control over non-power control decrease for smaller ranges. Another thing to take into consideration here is that the routing protocol used to generate these results (refer to Section V-B) chose the routes based on the minimum number of hops between source and destination so the best case for 802.11 is also employed in the power-controlled case (for unbiased comparison at the MAC level). A better choice for power controlled networks would to use a series of metrics that take into account number of hops, aggregate path power consumption (summed over the power required at each intermediate link), and spectral reuse gains that account for node densities in various areas of the network to make the best path decisions. This, however, is a topic that is outside of the focus of this discussion so we stay focused on the power control at the MAC layer and leave routing level power control issues for future research though we mentioned this so as to highlight the fact that additional benefits are still possible when utilizing transmission power controlled protocols.

Let us now distinguish between the two spectral reuse benefits specified in the proceeding two paragraphs. The first spectral reuse that is provided to both 802.11 and 


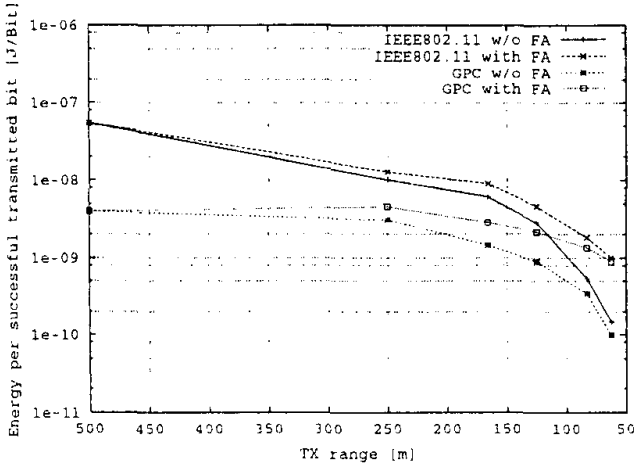

Fig. 7. Signal energy per successfully transmitted bits for uniform forwarding agent placement with different transmission ranges

GPC is due to the decrease in maximum transmission range and utilizing intermediate hops. This range dictates the number of intermediate hops that must be used between a given source-destination pair. The second additional spectral reuse benefit is from reducing (or controlling) the transmission power to that needed to reach the intended receiver (next hop) that must be chosen from the nodes within the fixed maximum range, which is varied between $500 \mathrm{~m}$ and $62.5 \mathrm{~m}$. The first case can only be realized in 802.11 if we manually adjust (restrict) the output power of all nodes, while a power-controlled MAC protocol (like the ones presented in [7], [6]) will dynamically adjust the power to the intended receiver so it would be enough to restrict the nodes that can be used as next hops in the routing protocol, without manually adjusting the transmission range. In accordance with the limitations of current non-power-controlled MAC protocols like 802.11 presented in Section II, it can be concluded that even if the transmission power levels of 802.11 can be dynamically adjusted to satisfy the intended destination it is still not sufficient since it would violate the collision avoidance framework set forth by the communal structure of shared channel ad hoc networks.

The next network topologies that are studied (Scenario II from Section IV) are those which designate a subset of the nodes as forwarding agents for a cluster of nodes (chosen either for strategic reasons (see [17]) or because they have a greater degree of resources). Nodes send directly to nodes in the same cluster, but go through the forwarding agent to send to nodes in other clusters.

For this type of network topology we start with the case where the forwarding agents positions can be controlled. A simple method would be to place some number of forwarding agents uniformally within the network area. However, instead of placing a specified number of forwarding agents in the network area, the maximum transmission range is specified and a minimum number of forwarding agents are then placed such that every location in the network is in range of a forwarding agents and every forwarding agent

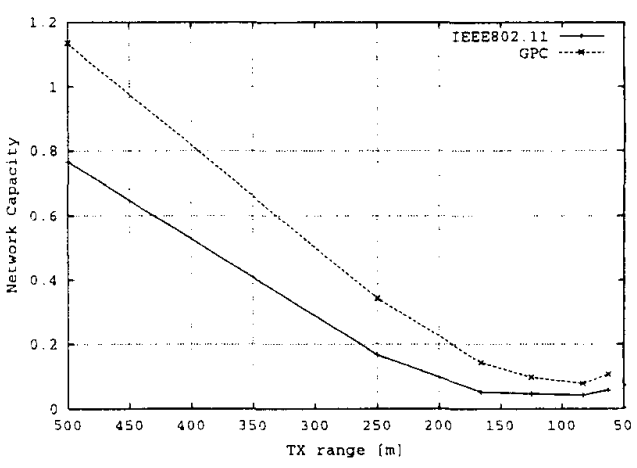

Fig. 8. Normalized throughput for uniform forwarding agent placement with different transmission ranges

is in range of its adjacent forwarding agent. The number of forwarding agents then required to cover the network area for the previously stated transmission ranges $(500$, $250,166,125,82$, and $62.5 \mathrm{~m}$ ) is then $1,4,9,16,25$, and 36 , respectively.

In Fig. 7, the signal energy per successfully transmitted bit is shown for both 802.11 and GPC for two different cases. The first case does not account for the energy consumed by the nodes designated as forwarding agents (assuming they have infinite resources such as a vehicle or node with a continuous supply of power; referred to as w/o FA in the graph). In the second case, the power of the forwarding agents is taken into account (they may have greater resources such as a laptop as compared to a sensor, but their power must still be considered; referred to as with FA in the graph). The results shown in the figure demonstrate again that there is a considerable energy savings as the transmission range is decreased and additional intermediate hops are utilized. Also as expected the power-controlled protocol saves energy over the non-power-controlled protocols though again the degree of benefit decreases as the maximum transmission range is decreased. The change in energy expenditure between the cases that account for the forwarding agent power consumption and those that do not start off small, but increases as the transmission range decreases since the number of forwarding nodes between source and destination increases as the range decreases. The results demonstrate that the energy consumed for the uniform placement of forwarding agents is less than the Scenario I with no infrastructure enhancements when the energy of the forwarding agents is not taken into account. Therefore, they are most desirable in heterogeneous networks where mobile nodes have a great variance in energy resources.

The throughput for this scenario is shown in Fig. 8 . It drops considerably more than for the previous scenario shown in Fig. 6. This is because all packets are forced to communicate through a single access point (forwarding agent) to all nodes in other clusters, and the number of 
nodes in other clusters increases as the range decreases because the cluster size decreases and there are more clusters. If we consider the point on the figure corresponding to the $250 \mathrm{~m}$ transmission range, we see that considerably less packets were delivered than for the infrastructureless case. This is because the forwarding agent employs the 802.11 distributed MAC algorithm which provides equal access rights to all nodes including those that were designated as the forwarding agent. Therefore, when $\mathrm{N}-1$ sources are all trying to send through a single forwarding agent that must relay the packets to $\mathrm{N}-1$ destinations, the one forwarding agent will be competing with the $\mathrm{N}-1$ sources to send to the $\mathrm{N}-1$ destinations, but only receive $1 / N$ share of the network resources. This will severely limit the number of packets that are allowed to reach the destinations, which is why the throughput is severely reduced when using the distributed 802.11 MAC with forwarding agents. As the number of forwarding agents is increased and the transmission range is reduced the rate initially declines because it takes more hops, but later starts to increase because the number of nodes the forwarding agent is competing with decreases.

The power-controlled protocol provides a considerable improvement when the greater transmission ranges are used, but deceases as the range is decreased since, similar to Scenario I, there is less spectral reuse to exploit.

The next figures are again for predesignate forwarding agents though this time the placement of these forwarding agents is random, Scenario III from Section IV. The motivation behind using forwarding agents under this scenario is that we desire to take advantage of nodes with greater resources as forwarders when they are within range. For each range the number of forwarding agents placed was made equal to the number of uniformly placed forwarding agents required to cover the graph for that corresponding range. Since the placement of the forwarding agents was not controlled and therefore random, it could not be guaranteed that all nodes are reachable from all other nodes. So as mentioned when discussing the simulation setup, the maximum transmission range for this topology scenario was kept constant at $500 \mathrm{~m}$, but the number of forwarding agents was set to $1,4,9,16,25$, and 36 . Then the $\mathrm{x}-$ axis corresponds to the transmission ranges shown in the previous figures. Note again that the transmission range shown in Fig. 4 corresponds to these number of forwarding agents for the random forwarding agent placement curve in this figure.

The signal energy used per successful bit for this scenario is shown in Fig. 9. The energy consumed by 802.11 actually increases as the number of forwarding agents increase. This is because the cluster size decreases on average with the number of forwarding agents, but the non-powercontrolled 802.11 protocol is not able to reduce its power to the size of the cluster distance to the forwarder. Although, GPC's energy consumption decreases because the power is reduced as the average distance to the forwarding agent

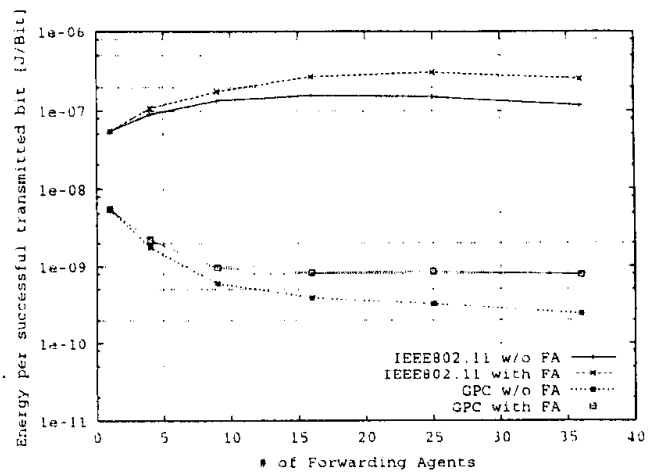

Fig. 9. Signal energy per successfully transmitted bit for random forwarding agent placement with different transmission ranges (\# of forwarding agents)

is reduced. Notice the energy consumed by both protocols changes at first dramatically because the average distance to the nearest forwarding agent decreases significantly as the first few are added, but the average distance to the forwarder decreases less significantly as the number of forwarders is further increased. For the last points the energy consumption decreases for both protocol (particularly those neglect the forwarding agents power consumption). This is due to the number of forwarding agents becoming a considerable number of the total sources such that the average hops decreases (see Fig. 4) without changing the distance? between forwarding agents, or forwarding agents and reg11lar nodes. Therefore the number of successful packets is increased without changing the average transmission power so the energy per successful bit drops. The energy consumed for the random placement of forwarding agents is more than for the uniform case even though the average distance between a node and its forwarding agent should be the same when many forwarders are present by the law of large numbers. However, the random case pays more heavily for a situation when the case where forwarders are further than the average than it gains when the forwarders are closer by the same amount since the gain is super linear (a convex function).

The throughput for the network with random placement of forwarding agents is shown in Fig. 10. The throughput for this scenario drops as the number of forwarding agents are increased. There are several factors contributing to this. First, as the number of forwarding agents is increased the cluster size decreases and therefore the number of nodes in the same cluster decreases, and the number of other clusters increases so a greater number of hops are required on average. In addition, the number of access points available for sending to other clusters is limited by the number of forwarding agents in range. Furthermore, as discussed in the uniform placement case, all the nodes in the cluster are contending with the forwarding agent such the number of packets that can be sent to other clusters is 


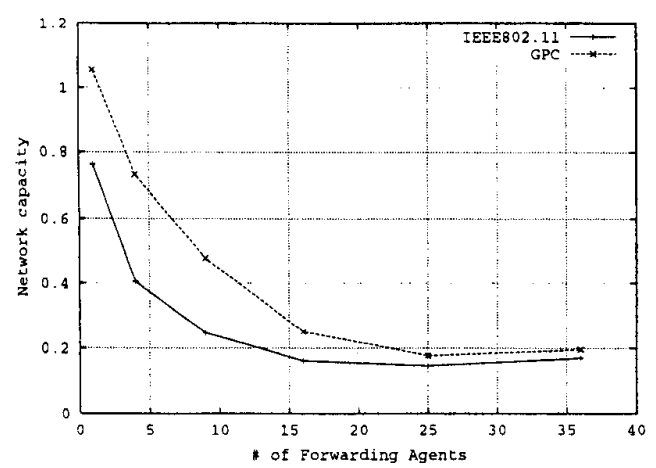

Fig. 10. Normalized throughput for random forwarding agent placement with different transmissions ranges (\# of forwarding agents)

limited by the nodes contending in the cluster. This effect becomes less significant as the number of nodes in the cluster decreases, but the dependence on the forwarding agent increases as the cluster size shrinks. These factors cause the throughput to continue to go down with increasing number forwarders, until the case where the forwarders become a considerable number of the total nodes and such that the average hops are suddenly reduced (refer to Fig. 4).

The throughput of the random placement case is better than that of the uniform placement case because the average hops are considerably less as can be observed in Fig. 4 . Finally, we observe that power control provides the greatest benefits over non-power-controlled protocols when the distance between source and destination are least limited and the average number of hops are small.

\section{SUMMARY}

This work evaluated the merits of power controlled MAC protocols in multi-hop wireless ad hoc type packet networks with respect to energy savings and throughput. We compared a generalized power control protocol (GPC) to a non-power controlled protocol (IEEE 802.11) in three different network setups which we believe are general to a certain extend.

It was shown that there are extensive benefits in energy savings by utilizing both intermediate nodes (between source and destination) and a power controlled protocol when sending between those hops. The throughput (capacity) was shown to increase when implementing power control. From this study it can be concluded that using power control can.always provide benefits.

Using a logical infrastructure with designated forwarding agents sending packets between clusters on single shared wireless data channel will gradually limit the capacity as the number of clusters is increased but improves the energy consumption performance. In addition, if forwarding agents can be utilized that do not have limited energy resources then the additional energy savings may further ju- stify their use. We also found, that the placement of forwarding agents for the cluster approach has been performed in a controlled manner to be most beneficial for the non-power-controlled case. Using shorter range transmissions with more hops provides considerable improvements in energy savings though it reduces throughput. Overall, the savings in energy for increasing the number of hops is far greater than the throughput we forgo. Finally, our results indicate that power control will be very beneficial in wireless environments as the distances between source and destination or forwarders change since the RF transmission power can be adapted to the range.

\section{REFERENCES}

[1] F. Rashid-Farrokhi and et. al., "Downlink power control and base station assignment," IEEE Communications Letters, vol 1, pp. 102-104, July 1997.

[2] N. Bambos, "Toward power-sensitive network architectures in wireless communications: Concepts, issues, and design aspects," IEEE Personal Communications, vol. 5, pp. 50-59, June 1998.

[3] P. Gupta and P. Kumar, "The capacity of wireless networks," IEEE Transactions on Information Theory, vol. IT-46, pp. 388404, March 2000.

[4] J. Monks, v. Bharghavan, and W. Hwu, "Transmission power control for multiple access wireless packet networks," IEEE Conference on Local Computer Networks LCN, vol. 25, pp. 1221, Nov. 2000.

[5] Jean-Pierre Ebert, Björn Stremmel, Eckhardt Wiederholt, and Adam Wolisz, "An Energy-efficient Power Control Approach for WLANs," Journal of Communications and Networks (JCN), vol. 2, no. 3, pp. 197-206, September 2000, Publication of Korean Institute of Communications Sciences (KICS).

[6] S. Wu, et. al., "Intelligent medium access for mobile ad hoc netowrks with busy tones and power control," IEEE Journal on select areas in communications, vol. 18, pp. 1647-57, Sept. 2000.

[7] J. Monks, V. Bharghavan, and W. Hwu, "A power controlled multiple access protocol for wireless packet networks," IEEE Conference on Computer Communications (INFOCOM), vol. 20, pp. 1-11, Apr. 2001.

[8] IEEE Std 802.11 - 1997, Wireless LAN Medium Access Control (MAC) and Physical Layer (PHY) Specifications, 1997.

[9] Intersil Corporation, "PRISM Chipset," http://www.intersil.com/design/prism/.

[10] Brian Burns and Jean-Pierre Ebert, "Power Consumption, Throughput and Packet Error Measurements of an IEEE 802.11 Interface," TKN Technical Report TKN-01-007, Telecommunication Networks Group, Technical University Berlin, http://www-tkn.ee.tu-berlin.de, August 2001.

[11] T. Rappaport, Wireless Communications: Principles and Practice, Prentice Hall, New Jersey, 1996.

[12] P. Karn, "MACA - a new channel access method for packet radio," ARRL/CRRL Amateur Radio 9th Computer Networking Conference, pp. 134-140, Sept. 1990.

[13] V. Bharghavan, A. Demers, S. Shenker, and L. Zhang, "MACAW: A media access protocol for wireless LAN's," Proceedings of ACM SIGCOMM, vol. 24, pp. 212-25, Oct. 1994.

[14] C. Fullmer and J. Garcia-Luna-Aceves, "Floor acquisition multiple access (FAMA) for packet-radio networks," Proceedings of ACM SIGCOMM, Sept. 1995.

[15] P. Johansson and et. al., "Scenario-based performance analysis of routing protocols for mobile ad-hoc networks," ACM International Conference on Mobile Computing and Networking (MOBICOM), pp. 195-206, 1999.

[16] "CMU Monarch Project," http://www.monarch.cs.cmu.edu/, Dec. 1999.

[17] Wendi Rabiner Heinzelman, A Chandrakasan, and Hari Balakrishnan, "Energy-efficient Communication Protocol for Wireless Microsensor Networks," in Proceedings of the 33rd International Conference on System Sciences (HICSS 'DO), January 2000. 